OPEN ACCESS

Edited by:

Senthil-Nathan Sengottayan,

Manonmaniam Sundaranar University,

India

Reviewed by:

Pin-Jun Wan,

Chinese Academy of Agricultural

Sciences, China

Yifan Zhai,

Shandong Academy of Agricultural

Sciences, China

${ }^{*}$ Correspondence:

Xunbing Huang

xunbingh@163.com

Specialty section:

This article was submitted to Invertebrate Physiology, a section of the journal

Frontiers in Physiology

Received: 22 September 2019

Accepted: 21 January 2020

Published: 21 February 2020

Citation:

Huang X, Lv S, Zhang Z and

Chang BH (2020) Phenotypic

and Transcriptomic Response of the

Grasshopper Oedaleus asiaticus

(Orthoptera: Acrididae) to Toxic Rutin.

Front. Physiol. 11:52

doi: 10.3389/fphys.2020.00052

\section{Phenotypic and Transcriptomic Response of the Grasshopper Oedaleus asiaticus (Orthoptera: Acrididae) to Toxic Rutin}

\author{
Xunbing Huang ${ }^{1 *}$, Shenjin $L v^{1}$, Zehua Zhang ${ }^{2}$ and Babar Hussain Chang ${ }^{2,3}$ \\ ${ }^{1}$ College of Agriculture and Forestry Science, Linyi University, Linyi, China, ${ }^{2}$ State Key Laboratory for Biology of Plant \\ Diseases and Insect Pests, Institute of Plant Protection, Chinese Academy of Agricultural Sciences, Beijing, China, \\ ${ }^{3}$ Department of Entomology, Sindh Agriculture University, Tando Jam, Pakistan
}

Rutin, a widely distributed phytochemical flavonoid, can be used to control insect pests. In this study, we studied the growth performance of the grasshopper Oedaleus asiaticus Bey-Bienko given xenobiotic rutin using feeding experiments and transcriptomic analysis. O. asiaticus had reduced body size, lower survival rate, and reduced growth performance when fed with xenobiotic rutin. Rutin-fed nymphs had large variation in gene expression profiles, with a total of 308 genes significantly upregulated and 287 genes downregulated. The upregulated genes were significantly enriched in stress resistance-, immune-, and detoxification-related biological processes and the Kyoto Encyclopedia of Genes and Genomes (KEGG) pathways. Downregulated genes mainly involved cuticle biosynthesis and nutrition metabolism-related pathways. The quantitative real-time PCR (qRT-PCR) analysis of 15 candidate genes also produced results consistent with the transcriptome data. These results suggested that grasshoppers' capacity for biosynthesis and nutrition metabolism decreased, and stress resistance and metabolized capacity to toxic substances were significantly induced when $O$. asiaticus was fed on xenobiotic rutin. Rutin, as a phytotoxin, had detrimental effects and induced changes in gene expression profiles for $O$. asiaticus. This study can provide a molecular basis and offer future opportunities for the development of rutin-related insecticides and their application to grasshopper control.

Keywords: grasshopper, rutin, phenotype, transcriptomics, biopesticides

\section{INTRODUCTION}

The use of synthetic pesticides undoubtedly has contributed to the development of agriculture, but it has also caused problems, including environmental toxicity and residual properties in water, soil, and crops (Senthil-Nathan, 2013; Jallow et al., 2017). In addition, the indiscriminate use of synthetic pesticides generally results in insect pest resistance and resurgence. The hazardous effects on the environment associated with pesticides can endanger the sustainability of ecosystems (Senthil-Nathan, 2013; Cevizci et al., 2015). Hence, searching for ecofriendly biopesticides that are target specific, rapidly degradable, and low in toxicity is essential. Secondary metabolites derived from plants, such as alkaloids, flavonoids, terpenoids, and sterols, are important resources for 
such biopesticides (De Oliveira et al., 2014; Isman, 2014; Campos et al., 2018). These chemical compounds normally rapidly degrade and, as such, lack persistence in the ecosystem (Theis and Lerdau, 2003; Senthil-Nathan, 2013; Michael, 2018; MonsrealCeballos et al., 2018). Research concerning the effects of these compounds on insect pests can offer future opportunities in the development of new bioinsecticides.

Generally, many plant-derived compounds can be used as toxicants, and they have been shown to have adverse effects to insect pests (Leicach and Chludil, 2014; Mouden et al., 2017; Michael, 2018). Diet stress from toxic exposure can significantly affect herbivorous insects. Some toxic compounds can cause mortality or reduced growth of pest insects through feeding deterrence or oviposition deterrence (Tangtrakulwanich and Reddy, 2014). For example, chemical azadirachtin can produce intestinal lesions, destroy digestive enzyme systems, and eventually lead to insect death (Ley, 2005; Senthil-Nathan, 2013). Nicotine, matrine, and quercetin-based biopesticides can have sublethal, lethal, or other deleterious effects on coleopteran and lepidopteran pest species (Senthil-Nathan, 2013; Poreddy et al., 2015).

Insect herbivores have evolved multiple strategies to overcome these plant chemical defenses, including contact and ingestion avoidance, sequestration, excretion, target-site mutation, and degradation of the toxin (Després et al., 2007; Poreddy et al., 2015; Birnbaum et al., 2017). The related molecular processes of detoxifying mechanisms, transporters, immunity, and peritrophic membranes allow insects to develop resistance to plant toxic substances (Roy et al., 2016; Birnbaum et al., 2017). For example, molecular adaptation has allowed more robustly active digestive, protective, and detoxifying-related enzymes in herbivorous insects; this includes the rapid synthesis of hydrolase, antioxidase, or cyP450s (Després et al., 2007; Senthil-Nathan, 2013). Such physiological responses to xenobiotic chemicals are vitally important for insect survival and reproduction (Roy et al., 2016).

Rutin, a widely distributed flavonoid glycoside, generally can help plants resist insect attack (Chen et al., 2017). The extracted flavonoid rutin from plants has deleterious effects on the pest insects Lymantria dispar, Spodoptera litura, Pectinophora gossypiella, Heliothis virescens, Spodoptera eridania, and Helicoverpa zea (Simmonds, 2003; Salunke et al., 2005; Mesbah et al., 2007; Taggar and Gill, 2016). Although insect phenotypic responses to toxic rutin are well documented, the underlying molecular basis of gene regulation are not well understood. Oedaleus asiaticus Bey-Bienko (Orthoptera: Acrididae), a dominant grasshopper pest of northern Asian grasslands, is distributed throughout northern China. Plagues of this grasshopper can severely damage grassland production and ecology and can aggravate grassland degradation (Cease et al., 2012; Zhang et al., 2013; Huang et al., 2016). Given the significant economic and ecological impact of this grasshopper, understanding the mechanism of grasshoppers' response to xenobiotic rutin can potentially improve management of the grasshopper.

In this study, we sought to reveal how xenobiotic rutin influences gene expression and the resulting phenotype of grasshopper $O$. asiaticus. Specifically, the transcriptomics underlying the manifold changes for toxic rutin were examined to explore the altered gene regulation. Our ultimate goal is to use such knowledge about phytochemicals and rutin-induced gene regulation to control insect pests.

\section{MATERIALS AND METHODS}

\section{Grasshopper Collection}

Third-instar nymphs of $O$. asiaticus were collected using sweep nets from Xilin Gol League $\left(43.968^{\circ} \mathrm{N}, 115.821^{\circ} \mathrm{E}\right)$, Inner Mongolia in northeastern China. These collected nymphs were maintained temporarily in metal frame cages $(50 \times 50 \times 50 \mathrm{~cm})$. Cages were then placed in an illumination incubator for 1 day under an artificial light regime ( $14 \mathrm{~h}$ light-10 h dark) at $28 \pm 1{ }^{\circ} \mathrm{C}$ and a relative humidity of $70 \pm 1 \%$. We used fresh-cut Stipa krylovii Roshev (the optimal food grass) to feed $O$. asiaticus nymphs until they were transferred to the feeding trial (see below).

\section{Feeding Trial}

We investigated $O$. asiaticus growth performance when the nymphs were reared using rutin-treated foods. Before the artificial rutin-feeding trial, a total of 400 third-instar female individuals were selected and starved for $12 \mathrm{~h}$ before the trial. Because the gender of the early instars is difficult to identify, third-instar female individuals were selected based on external morphology of the reproductive system. All selected nymphs were assigned to 20 plastic cages $(30 \times 20 \times 10 \mathrm{~cm})$, with each cage including 20 individuals. We used the grass S. krylovii, the most favored food of $O$. asiaticus, to feed the assigned nymphs. S. krylovii was collected freshly from the field and trimmed to $10 \mathrm{~cm}$, then treated with rutin. In previous studies, we found that $\sim 0.01 \%$ rutin content in food plant had significant adverse effects to O. asiaticus (Huang et al., 2017; Li et al., 2019). To further explore the grasshopper phenotypic and transcriptomic response to toxic rutin, we prepared $0.01 \%$ rutin solution using sterile water. For each cage, $100 \mathrm{ml}$ prepared rutin solution was applied evenly to $100 \mathrm{~g}$ of fresh S. krylovii, and then provided to the grasshoppers. S. krylovii treated with only sterile water was used as the control (no rutin treatment) and was provided to the allocated cage using the same methods as above. Each treatment was replicated 10 times. The treated fresh S. krylovii was replaced every $24 \mathrm{~h}$ for each cage. Grasshoppers were cultivated in cages under an artificial light regime ( $14 \mathrm{~h}$ light- $10 \mathrm{~h}$ dark) at a temperature of $28^{\circ} \mathrm{C}$ and relative humidity of $70 \%$. Before the artificial feeding trial, another $30 \mathrm{O}$. asiaticus third-instar females were euthanized by diethyl ether and dried at $90^{\circ} \mathrm{C}$ for $24 \mathrm{~h}$, after which they were individually weighed and the mean (third-instar body mass, milligram) determined to serve as baseline data. Once grasshopper nymphs were assigned to each cage, we inspected grasshopper survival and removed dead individuals daily. This artificial feeding trial lasted for 7 days. For each treatment, a total of 30 female nymphs were collected (three females per cage) on the seventh day, and the nymphs were then used for RNAseq. The remaining surviving individuals at the last day were 
also euthanized and dried using the same method as above to determine their dry body mass (milligram). The increase in body mass (milligram) was calculated by subtracting the basic thirdinstar body mass from the grasshopper body mass of the feeding trial. Survival rate (\%) was calculated by the number of surviving individuals on last day/number of initial third-instar individuals $(n=20)$. Growth rate $(\mathrm{mg} /$ day) was calculated by the body mass increase (milligram)/development time (7 days).

\section{RNA Preparation, Library Construction, and Transcriptome Sequencing}

We collected three samples from each of our two treatments at the seventh day of the feeding trial. Each sample consisted of 10 female nymphs (one chosen randomly from each of the 10 replicates). Hence, six samples (three biological replicates from each treatment) were analyzed. Each sample consisted of 10 female nymphs (combined). The collected samples were named by abbreviating the grasshopper name and treatment followed by the sample number; OA_CK-1, OA_CK-2, OA_CK-3, OA_ Rutin-1, OA_Rutin-2, and OA_Rutin-3.

We used TRIzol reagent (Invitrogen, CA, United States) to extract total RNA from each sample according to the manufacturer's instructions. The RNA sample quality was monitored on $1 \%$ agarose gels and checked by the NanoPhotometer spectrophotometer (IMPLEN, CA, United States), Qubit RNA Assay Kit in the Qubit 2.0 Fluorometer (Life Technologies, CA, United States), and RNA Nano 6000 Assay Kit of the Agilent Bioanalyzer 2100 system (Agilent Technologies, CA, United States). High-quality RNA $(1.9<\mathrm{OD} 260 / 280<2.1$; RNA integrity number $R I N>8.0)$ from each sample was used for complementary DNA (cDNA) library construction. Then, sequencing libraries were generated using the NEBNext Ultra RNA Library Prep Kit for Illumina (NEB, United States) following the manufacturer's recommendations. Finally, the libraries were sequenced on the Illumina HiSeqTM 4000 platform (Illumina Inc., San Diego, CA, United States).

\section{Data Processing and Differential Expression Analysis}

Raw reads in fastq format were first processed through an in-house perl script. Adapters and reads containing poly- $\mathrm{N}$ or of low quality were removed. Q20, Q30, GC content, and sequence duplication level of the clean data were used for data filtering. After filtering the raw reads, de novo assembly of the transcriptome was carried out using Trinity (Grabherr et al., 2011). Then, generated unigenes were used for BLASTX searches and annotated based on the following databases: NCBI non-redundant nucleotide database $(\mathrm{Nt})$, Eukaryotic Ortholog Groups (KOG), National Center for Biotechnology Information (NCBI) non-redundant protein database $(\mathrm{Nr})$, the Swiss-Prot protein database (Swiss-Prot), and the Kyoto Encyclopedia of Genes and Genomes (KEGG). All searches were performed with an $E$ value $<10^{-5}$. In addition, we used the Blast2GO program (Conesa et al., 2005) to obtain the Gene Ontology (GO) annotation of unigenes.
The sequenced reads for each sample were remapped to the assembled transcriptome using SOAPaligner/soap2 (Li et al., 2009). Gene expression values were quantified as fragments per kilobase per million mapped reads (FPKM) by RNA-Seq by Expectation Maximization (RSEM) (Li and Dewey, 2011). Differentially expressed genes (DEGs) were detected by DESeq2 $\mathrm{R}$ package (Anders and Huber, 2010). Count matrix was obtained using featureCounts function in the Rsubread package (Liao et al., 2013). Transcripts with a minimum 2-fold difference ( $\mid \log _{2}$.Fold_change $>1$ ) in expression and adjusted $P<0.05$ were considered differentially expressed between the control and rutin-treated group.

\section{Functional Analysis of DEGs}

Differentially expressed genes were annotated to the GO database by the GOseq $\mathrm{R}$ packages based on Wallenius non-central hypergeometric distribution (Young et al., 2010) and mapped to pathways in the KEGG database using KOBAS software (Mao et al., 2005). GO terms significantly enriched for DEGs were identified by hypergeometric tests using transcriptome background. The resulting $P$ values were adjusted using the Benjamini-Hochberg $(\mathrm{BH})$ method. A corrected $P<0.05$ was used as the threshold for statistical significance.

\section{Quantitative Real-Time PCR Validation}

Fifteen candidate DEGs (Supplementary Table S1) involved in grasshopper cuticle biosynthesis, nutrition metabolites, stress resistance, or detoxifying enzymes were chosen for validation using quantitative real-time PCR (qRT-PCR). These candidate genes were FOXO (FoxO protein, Cluster-11327.75652), HSP 90 (heat shock protein 90, Cluster-11327.59722), CYP450 6K1 (cytochrome P450 $6 \mathrm{~K} 1$, Cluster-11327.54882), CYP450 9E1 (cytochrome P450 9E1, Cluster-11327.70406), UGT 2C1 (UDPglucuronosyltransferase 2C1, Cluster-11327.22262), UGT $2 B 1$ (UDP-glucuronosyltransferase 2B1, Cluster-11327.24983), LPH (Lactase-phlorizin hydrolase, Cluster-11327.46783), BG (betaglucosidase, Cluster-11327.3892), SOD (superoxide dismutase, Cluster-11327.55763), POD (Peroxidase, Cluster-11327.56874), CarE (carboxylesterase, Cluster-11327.66426), VG (vitellogenin, Cluster-11327.56459), LCP (Larval cuticle protein 2, Cluster11327.87505), CS (Chitin synthase 1, Cluster-11327.80081), and $C P$ (Cuticle protein 7, Cluster-11327.93652).

Gene-specific primers (Supplementary Table S2) for qRTPCR of those 15 genes were designed using the software Primer $3^{1}$. We collected one nymph on the last day of the feeding trial randomly from each replicate cage of the two treatments (20 samples). Total RNA was extracted using the same method as described above. The cDNA was synthesized using avian myeloblastosis virus (AMV) reverse transcriptase (Invitrogen, Carlsbad, CA, United States) according to the manufacturer's instructions. The same qRT-PCR procedure described by Li et al. (2019) were used to detect the gene expression. Relative gene expression levels were analyzed using the $2^{-\Delta \Delta}$ CT method, with $\beta$-actin as reference gene (Zhang et al., 2015; Huang et al., 2017; Li et al., 2019). Expression values were adjusted by setting the

\footnotetext{
${ }^{1}$ http://bioinfo.ut.ee/primer3-0.4.0/
} 

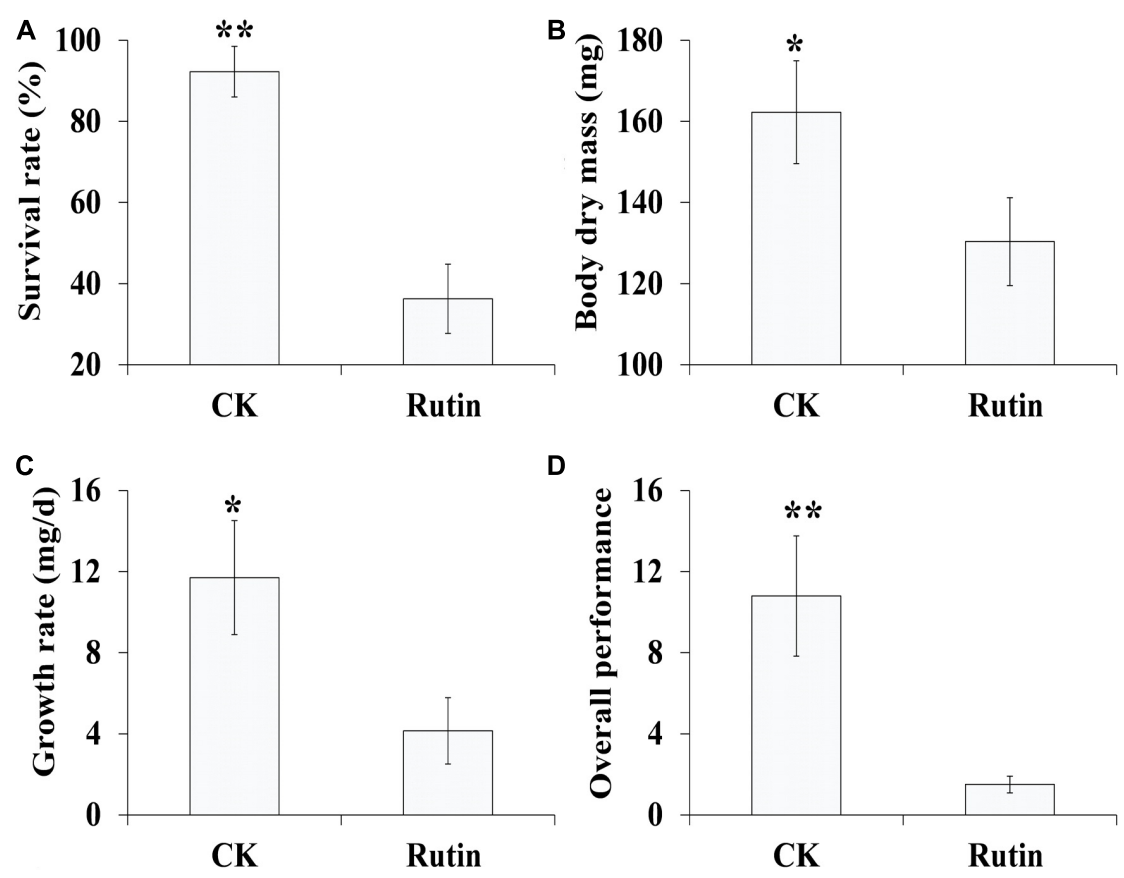

FIGURE 1 | (A) Oedaleus asiaticus mean\% survival rate $\pm \mathrm{SD}$, (B) mean dry mass (mg \pm SD), (C) mean growth rate (g/day \pm SD), and (D) overall performance $( \pm \mathrm{SD})$ when fed by rutin-treated foods. ${ }^{\star} P<0.05,{ }^{\star \star} P<0.01$ (Student's $t$ test).

expression of controls to be 1 for each gene. All methods and data collections in qRT-PCR followed the Minimum Information for Publication of Quantitative Real-Time PCR Experiments (MIQE) guidelines. All qRT-PCRs for each gene of 20 samples (10 biological replicates for each treatment) used three technical replicates per experiment.

\section{Data Analysis}

Student's $t$ test were used to compare grasshopper growth performance variables (survival rate and growth rate) in feeding trial, and the relative gene expression by qRT-PCR. SAS version 8.0 were used for all analyses.

\section{RESULTS}

\section{Growth Performance of 0 . asiaticus to Xenobiotic Rutin}

In the artificial feeding trial, we examined O. asiaticus' growth performance in response to xenobiotic rutin (Figure 1). O. asiaticus feeding on xenobiotic rutin had significantly reduced survival rate (Figure 1A), body mass (Figure 1B), growth rate (Figure 1C), and overall performance (Figure 1D) compared to $\mathrm{CK}$. This indicates that feeding on rutin has detrimental effects for O. asiaticus growth and development.

\section{Transcriptome Analysis}

To investigate the response of grasshoppers to xenobiotic rutin, the collected samples (three biological replicates) were analyzed by RNA-seq. Sequencing the transcriptomes of $O$. asiaticus fed on rutin generated 174,065,253 bases (Table 1). All six libraries were good quality, with Q20 and Q30 values >95 and $90 \%$, respectively, with a reasonable GC content (Table 1 and Supplementary Table S3). The total numbers of unigenes was 133,144 , with a mean length of 1,037 bp (Table 1 ).

Of the 133,144 unigenes, a total of 67,101 (50.39\%) were annotated in at least one database. Among these, 54,162 $(40.67 \%)$ were successfully annotated by NCBI Nr, 17,040 (28.14\%) by Swiss-Prot, 45,846 (34.43\%) by GO, 19.392 (14.56\%) by KEGG, 26,428 (19.84\%) by KOG, and 17,040 (12.79\%) by NCBI Nt (Supplementary Table S4). Transcriptome data have been submitted to the NCBI SRA database (accession number SRP072969). The majority of the sequences matched insect proteins, with the most abundant matching Zootermopsis nevadensis (30.6\%), Stegodyphus mimosarum (4.8\%), Tribolium castaneum (5.5\%), Acyrthosiphon pisum (3.2\%), and Lasius niger (2.8\%) (Supplementary Figure S1).

TABLE 1 | Statistics for the assembled sequences.

\begin{tabular}{lc}
\hline Group name & Number \\
\hline Total assembled bases & $174,065,253$ \\
Total number of unigenes & 133,144 \\
GC percentage (\%) & 46.68 \\
Unigene N50 (bp) & 1,705 \\
Unigene N90 (bp) & 533 \\
Maximum unigene length (bp) & 28,340 \\
Minimum unigene length (bp) & 2,250 \\
Average unigene length (bp) & 1,307
\end{tabular}




\section{Differentially Expressed Genes}

Differentially expressed genes (adjusted $P<0.01$, $\mid \log _{2}$.Fold_change $\mid>1$ ) between the control and rutinchallenged libraries were identified. Compared with controls, $O$. asiaticus feeding on rutin had 308 upregulated genes and 287 downregulated genes (Figure 2 and Supplementary Tables S5, S6).

Among the upregulated genes (Supplementary Table S5), many stress-resistant or detoxifying enzymes were identified, including cytochrome P450 9E1, heat shock protein, cytochrome P450 6k1, beta-glucosidase, catalase, carboxylesterase, peroxidase, superoxide dismutase, UDP-glucuronosyltransferase, and lactase-phlorizin hydrolase. This suggested that feeding on rutin could induce the expression of stress-resistance- and detoxification-related genes in the grasshopper O. asiaticus.

The downregulated genes mainly belonged to insect cuticle biosynthesis, development, and nutrition metabolism; these included vitellogenin, larval cuticle protein 2 , chitin synthase 1 variant $B$, cuticle protein 6 , hexokinase type 2 , and lipase 3 (Supplementary Table S6).

\section{GO and KEGG Pathway Enrichment Analysis of DEGs}

By the GOseq R package, the DEGs were mainly assigned to 15 GO terms (corrected $P<0.05$ ) (Figure 3 and Supplementary Table S7). Downregulated GO terms included oxidationreduction process, starch metabolic process, sucrose metabolic process, DNA-dependent DNA replication, structural constituent of cuticle, digestion, and growth factor binding. Upregulated GO terms included response to oxidative stress, regulation

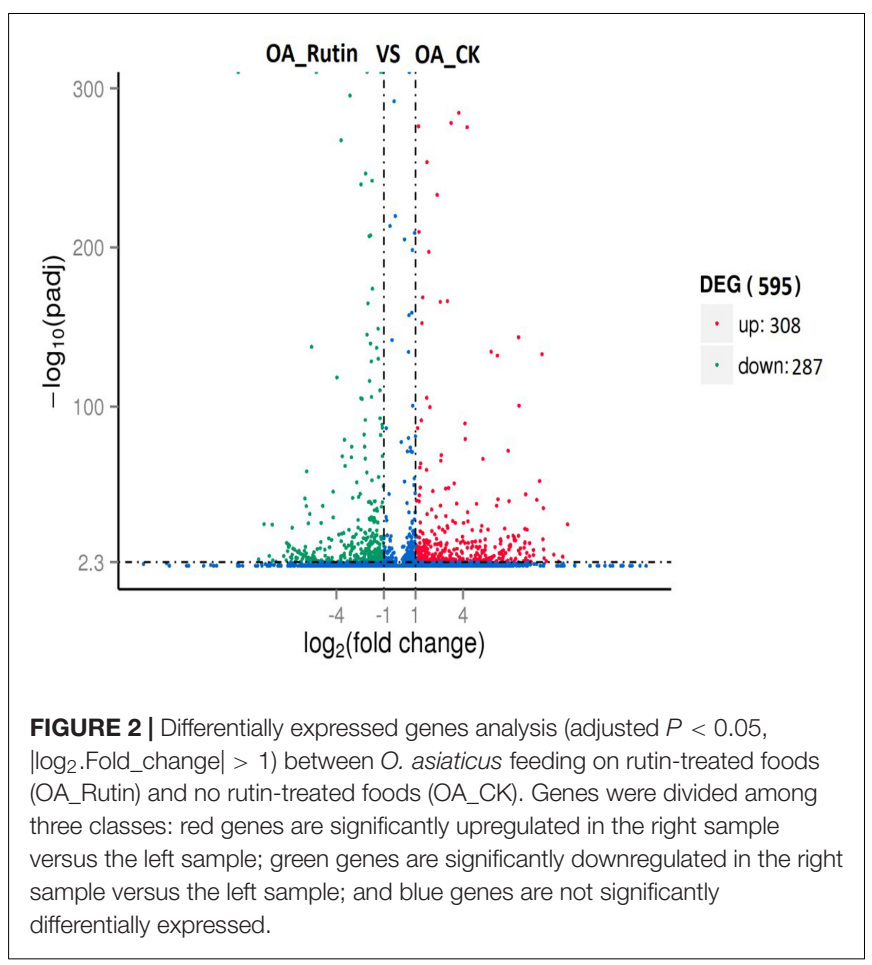

of signal transduction, defense response, response to toxic substance, regulation of immune system process, xenobiotic metabolic process and antioxidant activity, and cellular response to chemical stimulus. This suggests that $O$. asiaticus feeding on xenobiotic rutin had increased signal transduction, stress resistance, and metabolizing capacity of toxic substances.

The DEGs were mainly assigned to 14 (corrected $P<0.05$ ) pathways (Figure 4 and Supplementary Table S8). Downregulated pathways included N-glycan biosynthesis, nitrogen metabolism, carbohydrate digestion and absorption, oxidative phosphorylation, fat digestion and absorption, insulin signaling pathway, and protein digestion and absorption. Upregulated pathways included metabolism of xenobiotics by cytochrome P450, hypoxia-inducible factor 1 (HIF-1) signaling pathway, FoxO signaling pathway, Jak-STAT signaling pathway, AMP-activated protein kinase (AMPK) signaling pathway, peroxisome, and apoptosis. This suggests that rutin-fed $O$. asiaticus had increased activities in detoxification, immune response, and stress resistance.

\section{Verification of the Gene Expression Through qRT-PCR}

Relative gene expression of 15 candidate genes were tested by qRT-PCR. Results showed that the metabolism- or cuticle biosynthesis-related genes $V G, L C P, C S$, and $C P$ were significantly downregulated in $O$. asiaticus fed on rutin $(P<0.05$, Figure 5). In contrast, the stress-resistance- or detoxificationrelated genes FOXO, HSP 90, CYP450 6K1, CYP450 9E1, UGT 2C1, UGT 2B1,LPH, BG, SOD, POD, and CarE were significantly upregulated $(P<0.05$, Figure 5). Moreover, 15 genes showed significant correlations $\left(R^{2}=0.8937, P<0.05\right)$ between the qRT-PCR data and the RNA-seq results, which indicated good reproducibility between transcript abundance assayed by RNA-seq and the expression profile revealed by the qRT-PCR data.

\section{DISCUSSION}

In our experiment, we employed a feeding trial and RNA-seq to compare the suitability of xenobiotic rutin for the grasshopper $O$. asiaticus. The results demonstrate that xenobiotic rutin, as a phytotoxin, is detrimental for $O$. asiaticus. Grasshoppers fed on rutin-treated food had reduced size, growth, and survival rate. These results were consistent with those from previous research concerning the effects of flavonoid rutin on other insects (Simmonds, 2003; Taggar and Gill, 2016; Chen et al., 2017) and supported the hypothesis that insect growth performance was negatively correlated with plant toxic compounds. Subsequent transcriptomic analysis demonstrated that rutin-fed grasshoppers exhibited dramatically different transcription profiles from control grasshoppers. Why would feeding on rutin alter transcription profiles, and what are the consequences of such changes? These are all important questions that need to be addressed.

Understanding the genetic and molecular basis of insect response mechanisms to chemical pressures (e.g., plant 

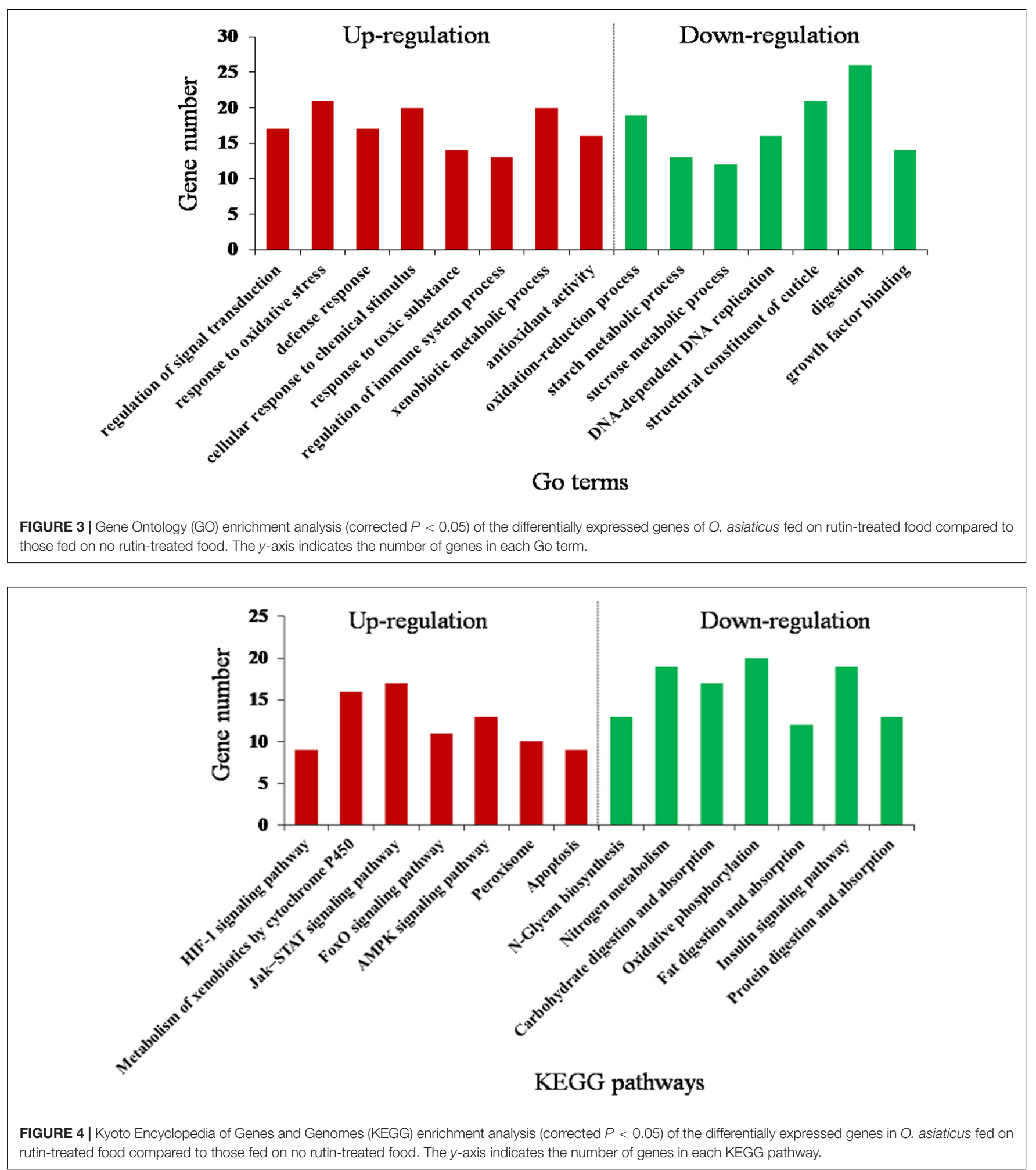

allelochemicals and other toxic compounds) is a key challenge in developing new pest control strategies. Toxic compounds in environment pose a constant challenge to the survival of insect pests (Després et al., 2007; Caballero et al., 2008; Chen et al., 2017). These toxins originating from a wide range of sources, such as plant toxins or chemical pesticides, generally have detrimental effects on insect pests (Senthil-Nathan, 2013; Birnbaum et al., 2017). To avoid these poisonous effects and sustain survival and reproduction, insects have evolved multiple strategies to overcome these chemical toxins, mainly involving 


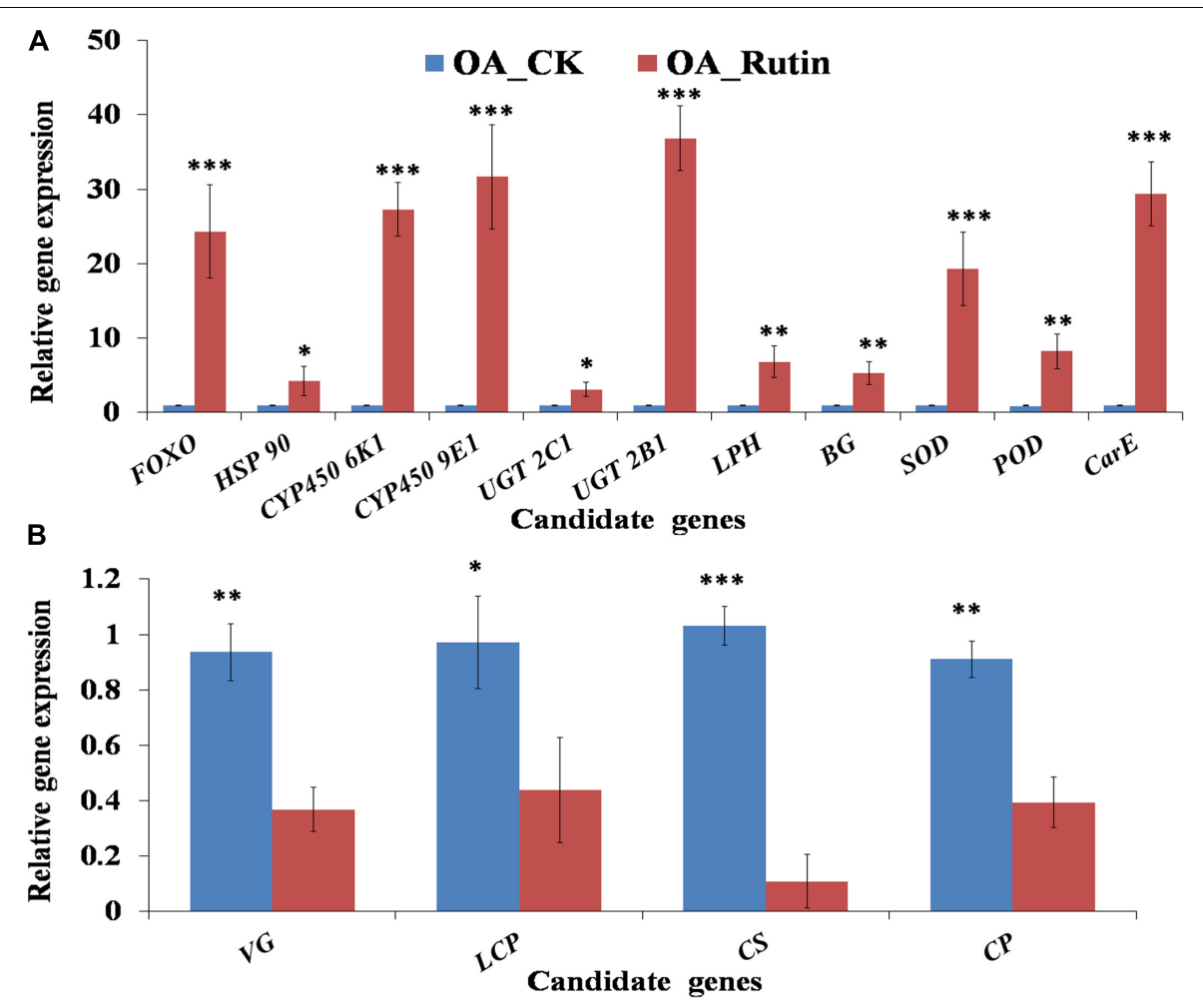

FIGURE 5 | Quantitative real-time PCR (qRT-PCR) analysis of 15 candidate genes. (A) Significantly up-regulated candidate genes in grasshoppers fed on rutin, (B) Significantly down-regulated candidate genes in grasshoppers fed on rutin. The results were evaluated using the $2^{-\Delta} \Delta$ CT method. The $2^{-\Delta} \Delta$ CT values of controls were set to one to calibrate the relative gene expression levels. Bars represent mean $\pm \mathrm{SD}$ values. ${ }^{*} P<0.05,{ }^{* *} P<0.01$, ${ }^{* * *} P<0.001$ (Student's $t$ test).

the complex induction of transporters, digestion, detoxification, or immune-related genes or pathways (Misra et al., 2011; SenthilNathan, 2013; Herde and Howe, 2014; Roy et al., 2016). In the present study, we found that these related genes and pathways were also significantly changed in grasshoppers feeding on rutin. Rutin induced 308 upregulated and 287 downregulated genes in $O$. asiaticus. Rutin-fed grasshoppers had increased activities in detoxification, immune, and stress resistance, and had decreased biosynthesis and nutrition metabolism.

Research over the past several decades has shown that the molecular adaptations of herbivorous insects to plant toxins mainly involve toxin degradation (metabolic adaptation) and target-site mutations (Salunke et al., 2005; Sun et al., 2006; Misra et al., 2011). Generally, metabolic adaptations of insects, in the most common strategy via biotransformation of plant toxins, include phases I, II, and III mechanisms (Després et al., 2007; Birnbaum and Abbot, 2018). These three metabolic mechanisms are involved in many detoxification enzymes, such as cytochrome P450s, carboxylesterases, glutathione-S-transferases (GSTs), and uridine diphosphate (UDP)-glycosyltransferases (UGTs). Through these complex processes, insects can modify the ingested chemical toxins and render them less toxic and easier to transport or excrete (Chahine and O'Donnell, 2011; Dobler et al., 2011). In the present study, grasshoppers feeding on rutin also had significantly upregulated gene expression for cytochrome P450 6k1, cytochrome P450 9E1, carboxylesterase,
UDP-glucuronosyltransferase 2B1, UDP-glucuronosyltransferase $2 \mathrm{C} 1$, and glutathione-S-transferases. These detoxification enzymes of grasshoppers may be significantly activated to enhance their catalytic activity toward toxic rutin. Aside from metabolic adaptations, many insect species have evolved "target-site modifications" at the binding location of toxins to protect themselves from plant toxic compounds (Després et al., 2007; Zhen et al., 2012; Ujvari et al., 2015). In addition, some gut microbes in insects can play important roles in the detoxification of toxic compounds (Henry et al., 2013; Giron et al., 2017; Birnbaum and Abbot, 2018). However, whether these mechanisms were also involved in the resistance of grasshoppers to rutin should be studied in the future.

In regard to the other specific DEGs for qRT-PCR, feeding on rutin resulted in downregulation of vitellogenin, larval cuticle protein, chitin synthase, and cuticle protein. These genes are related to insect development and cuticle biosynthesis. The upregulation of genes included heat shock protein, FoxO protein, peroxidase, and superoxide dismutase. These upregulated genes are related with insect stress resistance and oxidation-reduction and well known to be highly inducible and to ameliorate stress (Hwangbo et al., 2004; Krishnan and Kodrík, 2006; Lehtinen et al., 2006; Després et al., 2007; Tang et al., 2013). In addition, the upregulation of these genes is clearly beneficial for grasshoppers' survival. In contrast, we do not know if the downregulated genes are detrimental, beneficial, or simply accidental by-products of 
rutin stress. These rapid biochemical responses to toxic rutin may be vital for insect survival and growth. The reasons why these genes were downregulated need further study.

We acknowledge that, although the potential functions of some DEGs were discussed in the present study, more lab evidences should be provided to characterize their roles in response to rutin in the future. For example, we found that the gene expression levels of beta-glucosidase and lactase-phlorizin hydrolase were significantly upregulated. In mammals, these two enzymes can participate in the hydrolysis process of flavonoids by cutting off the glucosidic bond (Poreddy et al., 2015; Kohl et al., 2016). If these two hydrolases also participated in the hydrolysis of rutin in grasshoppers, it could be studied by gene technology (e.g., HIF-1) in the future.

From the GO and KEGG enrichment of DEGs, we found that the structural constituents to cuticle production and nutrition metabolic processes were significantly downregulated, and the pathways related to detoxification, stress resistance, and immunity (e.g., the Jak-STAT signaling pathway, metabolism of xenobiotics by cytochrome P450, and the FoxO signaling pathway) were significantly upregulated in rutin-fed grasshoppers. The fact that $O$. asiaticus upregulated stress resistance genes or pathways after feeding on rutin is not surprising, given that rutin is a toxic compound (Salunke et al., 2005; Kramer et al., 2008; Chen et al., 2017).

The altered transcriptome offers insights into the observed growth performance of grasshoppers exposed to rutin. Changes in phenotype indicate that the grasshoppers exhibit phenotypic plasticity when confronted with toxic rutin. Any organism can undergo such phenotypic plasticity, which can be expressed as changes in morphology or physiology (West-Eberhard, 2003; Whitman and Ananthrakrishnan, 2009). Many of these changes are probably responses to toxic stress, as indicated by the significantly lower growth performance observed in this study. Indeed, transcription underlies most phenotypic plasticity (Valentino and Harrelson, 2013). Small changes in transcription generally can produce significant changes to phenotypes. In this study, the changed transcription represented grasshoppers' phenotypic plasticity because it altered the phenotype (growth performance). Altered transcription responding to environmental stress can range from highly evolved and beneficial responses to non-evolved responses and consequently may have beneficial, neutral, or detrimental effects to living organisms (Whitman and Ananthrakrishnan, 2009; Valentino and Harrelson, 2013). Sorting out those effects from altered transcription is difficult. For example, transcriptomic changes of a single enzyme may influence numerous other enzymes or molecular pathways and consequently alter numerous divergent morphological and physiological aspects. Some of these changes may be beneficial, while others may produce detrimental effects (DeWitt and Scheiner, 2004; Whitman and Ananthrakrishnan, 2009). An example is the transcriptional changes delaying growth or reproduction; these may at first appear to be detrimental but in fact may be beneficial if they allow the individual to survive during a period of stress, such as during poisoning in the present study. In addition, the molecular or physiological mechanisms insects use to detoxify toxins as described above are generally assumed to be costly (Després et al., 2007). Costs of toxin resistance, such as reduced survival, fecundity, or energy reserves, have been demonstrated in many insect species (Rivero et al., 2011; Gordon et al., 2015; Schwenke et al., 2016). In the present study, grasshoppers feeding on rutin had higher levels of gene expression associated with detoxification or immunity, which implies that grasshopper survival requires greater consumption to detoxify rutin and consequently results in reduced phenotypic parameters such as size and growth rate compared to grasshoppers feeding on untreated foods.

One broad conclusion can be drawn from this study. Plant toxic compounds induce changed gene expression profiles of herbivorous insects. This confirms the hypothesis that altered transcription is related to environmental stress (Enders et al., 2015; Roy et al., 2016). Toxic stress substantially alters insect gene expression. In the present study, rutin-fed grasshopper exhibited 595 DEGs in comparison to untreated grasshoppers. In our case, we know that grasshoppers feeding on rutin were stressed because the feeding significantly lowered insect performance.

We explored phenotypic and transcriptomic responses in herbivores to toxic rutin using "Omics" technologies and found that rutin had detrimental effects to grasshoppers. This can offer future opportunities in the development of rutin-related insecticides for grasshopper control. O. asiaticus, as an important grassland pest, can cause severe grassland damage and lead to large economic losses in northern China (Cease et al., 2012). Traditional control of this grasshopper species relies too much on synthetic pesticides. To help reduce the occurrence of this disaster in an ecofriendly manner, the use of botanical pesticides is of great significance. In the future, we can develop and apply rutin-related insecticides or use rutin as the adjuvant to achieve new breakthroughs in grasshopper control.

\section{DATA AVAILABILITY STATEMENT}

The datasets generated for this study can be found in the NCBI, accession number SRP072969.

\section{ETHICS STATEMENT}

Grasshoppers Oedaleus asiaticus are common agricultural pests and are not included in the "List of Protected Animals in China." No specific permits were required for the described studies.

\section{AUTHOR CONTRIBUTIONS}

$\mathrm{XH}$ designed the experiments. $\mathrm{XH}, \mathrm{SL}$, and $\mathrm{BC}$ performed the experiments and wrote the manuscript. $\mathrm{XH}, \mathrm{BC}$, and $\mathrm{ZZ}$ analyzed the data. All authors reviewed and considered the manuscript.

\section{FUNDING}

This study was supported by the Shandong Provincial Natural Science Foundation, China, ZR2019BC030, National Natural 
Science Foundation of China, 31471823, and the Forage Industrial Innovation Team, Shandong Modern Agricultural Industrial and Technical System, China, SDAIT-23-10.

\section{ACKNOWLEDGMENTS}

We thank LetPub (www.letpub.com) for its linguistic assistance during the preparation of this manuscript and the reviewers

\section{REFERENCES}

Anders, S., and Huber, W. (2010). Differential expression analysis for sequence count data. Genome Biol. 11:R106. doi: 10.1186/gb-2010-11-10-r106

Birnbaum, S. S. L., and Abbot, P. (2018). Insect adaptations toward plant toxins in milkweed-herbivores systems-a review. Entomol. Exp. Appl. 166, 357-366. doi: 10.1111/eea.12659

Birnbaum, S. S. L., Rinker, D. C., Gerardo, N. M., and Abbot, P. (2017). Transcriptional profile and differential fitness in a specialist milkweed insect across host plants varying in toxicity. Mol. Ecol. 26, 6742-6761. doi: 10.1111/ mec. 14401

Caballero, C., López-Olguín, J., Ruiz, M., Ortego, F., and Castañera, P. (2008). Antifeedant activity and effects of terpenoids on detoxication enzymes of the beet armyworm, Spodoptera exigua (Hübner). Span. J. Agric. Res. 6, 177-184. doi: 10.5424/sjar/200806S1-386

Campos, E. V. R., Proença, P. L. F., Oliveira, J. L., Bakshi, M., Abhilash, P. C., and Fraceto, L. F. (2018). Use of botanical insecticides for sustainable agriculture: future perspectives. Ecol. Indic. 105, 483-495. doi: 10.1016/j.ecolind.2018. 04.038

Cease, A. J., Elser, J. J., Ford, C. F., Hao, S. G., Kang, L., and Harrison, J. F. (2012). Heavy livestock grazing promotes locust outbreaks by lowering plant nitrogen content. Science 335, 467-469. doi: 10.1126/science.1214433

Cevizci, S., Babaoglu, U. T., and Bakar, C. (2015). Evaluating pesticide use and safety practices among farmworkers in gallipoli peninsula, Turkey. Southeast Asian J. Trop. Med. Public Health 46, 143-154.

Chahine, S., and O'Donnell, M. J. (2011). Interactions between detoxification mechanisms and excretion in Malpighian tubules of Drosophila melanogaster. J. Exp. Biol. 214, 462-468. doi: 10.1242/jeb.048884

Chen, C., Han, P., Yan, W., Wang, S., Shi, X., Zhou, X., et al. (2017). Uptake of quercetin reduces larval sensitivity to lambda-cyhalothrin in Helicoverpa armigera. J. Pest Sci. 91, 919-926. doi: 10.1007/s10340-017-0933-1

Conesa, A., Gotz, S., Garcia-Gomez, J. M., Terol, J., Talon, M., and Robles, M. (2005). Blast2GO: a universal tool for annotation, visualization and analysis in functional genomics research. Bioinformatics 21, 3674-3676. doi: 10.1093/ bioinformatics/bti610

De Oliveira, J. L., Campos, E. V. R., Bakshi, M., Abhilash, P. C., and Fraceto, L. F. (2014). Application of nanotechnology for the encapsulation of botanical insecticides for sustainable agriculture: prospects and promises. Biotechnol. Adv. 32, 1550-1561. doi: 10.1016/j.biotechadv.2014.10.010

Després, L., David, J. P., and Gallet, C. (2007). The evolutionary ecology of insect resistance to plant chemicals. Trends Ecol. Evol. 22, 298-307. doi: 10.1016/j.tree. 2007.02.010

DeWitt, T. J., and Scheiner, S. M. (2004). Phenotypic Plasticity: Functional and Conceptual Approaches. Oxford: Oxford University Press.

Dobler, S., Petschenka, G., and Pankoke, H. (2011). Coping with toxic plant compounds-the insect's perspective on iridoid glycosides and cardenolides. Phytochemistry 72, 1593-1604. doi: 10.1016/j.phytochem.2011.04.015

Enders, L. S., Bickel, R. D., Brisson, J. A., Heng-Moss, T. M., Siegfried, B. D., Zera, A. J., et al. (2015). Abiotic and biotic stressors causing equivalent mortality induce highly variable transcriptional responses in the soybean aphid. G3 - Genes Genom. Genet. 5, 261-270. doi: 10.1534/g3.114.01 5149

Giron, D., Dedeine, F., Dubreuil, G., Huguet, E., Mouton, L., Outreman, Y., et al. (2017). Influence of microbial symbionts on plant-insect interactions. Adv. Bot. Res. 81, 225-257. doi: 10.1016/bs.abr.2016.09.007 for valuable suggestions to improve an earlier draft of this manuscript.

\section{SUPPLEMENTARY MATERIAL}

The Supplementary Material for this article can be found online at: https://www.frontiersin.org/articles/10.3389/fphys. 2020.00052/full\#supplementary-material

Gordon, J. R., Potter, M. F., and Haynes, K. F. (2015). Insecticide resistance in the bed bug comes with a cost. Sci. Rep. 5, 1-7. doi: 10.1038/srep10807

Grabherr, M. G., Haas, B. J., Yassour, M., Levin, J. Z., Thompson, D. A., Amit, I., et al. (2011). Full-length transcriptome assembly from RNA-Seq data without a reference genome. Nat. Biotechnol. 29, 644-652. doi: 10.1038/nbt.1883

Henry, L. M., Peccoud, J., Simon, J. C., Hadfield, J. D., Maiden, M. J. C., Ferrari, J., et al. (2013). Horizontally transmitted symbionts and host colonization of ecological niches. Curr. Biol. 23, 1713-1717. doi: 10.1016/j.cub.2013.07.029

Herde, M., and Howe, G. A. (2014). Host plant-specific remodeling of midgut physiology in the generalist insect herbivore Trichoplusia ni. Insect Biochem. Mol. Biol. 50, 58-67. doi: 10.1016/j.ibmb.2014.03.013

Huang, X., Ma, J., Qin, X., Tu, X., Cao, G., Wang, G., et al. (2017). Biology, physiology and gene expression of grasshopper Oedaleus asiaticus exposed to diet stress from plant secondary compounds. Sci. Rep. 7:8655. doi: 10.1038/ s41598-017-09277-z

Huang, X., Mcneill, M. R., and Zhang, Z. (2016). Quantitative analysis of plant consumption and preference by Oedaleus asiaticus (Acrididae: Oedipodinae) in changed plant communities consisting of three grasss pecies. Environ. Entomol. 45, 163-170. doi: 10.1093/ee/nvv172

Hwangbo, D. S., Gershman, B., Tu, M. P., Palmer, M., and Tatar, M. (2004). Drosophila dFOXO controls lifespan and regulates insulin signalling in brain and fat body. Nature 429, 562-566. doi: 10.1038/nature02549

Isman, M. B. (2014). Botanical insecticides: a global perspective. ACS Symp. Ser. 1172, 21-30. doi: 10.1021/bk-2014-1172.ch002

Jallow, M. F., Awadh, D. G., Albaho, M. S., Devi, V. Y., and Thomas, B. M. (2017). Pesticide knowledge and safety practices among farm workers in Kuwait: results of a survey. Int. J. Environ. Res. Public Health 14:E340. doi: 10.3390/ ijerph14040340

Kohl, K. D., Samuni-Blank, M., Lymberakis, P., Kurnath, P., Izhaki, I., Arad, Z., et al. (2016). Effects of fruit toxins on intestinal and microbial $\beta$-glucosidase activities of seed-predating and seed-dispersing rodents (Acomys spp.). Physiol. Biochem. Zool. 89, 198-205. doi: 10.1086/685546

Kramer, J. M., Slade, J. D., and Staveley, B. E. (2008). Foxo is required for resistance to amino acid starvation in Drosophila. Genome 51, 668-672. doi: 10.1139/G0 8-047

Krishnan, N., and Kodrík, D. (2006). Antioxidant enzymes in Spodoptera littoralis (Boisduval): are they enhanced to protect gut tissues during oxidative stress? J. Insect Physiol. 52, 11-20. doi: 10.1016/j.jinsphys.2005.08.009

Lehtinen, M. K., Yuan, Z., Boag, P. R., Yang, Y., Villén, J., Becker, E. B., et al. (2006). A conserved MST-FOXO signaling pathway mediates oxidative-stress responses and extends life span. Cell 125, 987-1001. doi: 10.1016/j.cell.2006. 03.046

Leicach, S. R., and Chludil, H. D. (2014). Plant secondary metabolites: structureactivity relationships in human health prevention and treatment of common diseases. Stud. Nat. Prod. Chem. 42, 267-304. doi: 10.1016/B978-0-444-632814.00009-4

Ley, S. V. (2005). Development of methods suitable for natural product synthesis: the azadirachtin story. Pure Appl. Chem. 77, 1115-1130. doi: 10.1351/ pac200577071115

Li, B., and Dewey, C. (2011). RSEM: accurate transcript quantification from RNASeq data with or without a reference genome. BMC Bioinformatics 12:323. doi: 10.1186/1471-2105-12-323

Li, R., Yu, C., Li, Y., Lam, T. W., Yiu, S. M., Kristiansen, K., et al. (2009). SOAP2: an improved ultrafast tool for short read alignment. Bioinformatics 25, 1966-1967. doi: 10.1093/bioinformatics/btp336 
Li, S., Huang, X., McNeill, M. R., Liu, W., Tu, X., Ma, J., et al. (2019). Dietary stress from plant secondary metabolites contributes to grasshopper (Oedaleus asiaticus) migration or plague by regulating insect insulin-like signaling pathway. Front. Physiol. 10:531. doi: 10.3389/fphys.2019.00531

Liao, Y., Smyth, G. K., and Shi, W. (2013). featurecounts: an efficient general purpose program for assigning sequence reads to genomic features. Bioinformatics 30, 923-930. doi: 10.1093/bioinformatics/btt656

Mao, X., Cai, T., Olyarchuk, J. G., and Wei, L. (2005). Automated genome annotation and pathway identification using the KEGG Orthology (KO) as a controlled vocabulary. Bioinformatics 21, 3787-3793. doi: 10.1093/ bioinformatics/bti430

Mesbah, H. A., Saad, A. S. A., Mourad, A. K., Taman, F. A., and Mohamed, I. B. (2007). Biological performance of quercetin on the cotton leaf-worm larvae, Spodoptera littoralis Boisd. (Lep. Noctuidae) and prevailing natural enemies in the Egyptian cotton fields. Commun. Agric. Appl. Biol. Sci. 72, 611-622.

Michael, W. (2018). Plant secondary metabolites modulate insect behavior-steps toward addiction? Front. Physiol. 9:364. doi: 10.3389/fphys.2018.00364

Misra, J. R., Horner, M. A., Lam, G., and Thummel, C. S. (2011). Transcriptional regulation of xenobiotic detoxification in Drosophila. Genes Dev. 25, 1796-1806. doi: 10.1101/gad.17280911

Monsreal-Ceballos, R. J., Ruiz-Sánchez, E., Ballina-Gómez, H. S., Reyes-Ramírez, A., and González-Moreno, A. (2018). Effects of botanical insecticides on hymenopteran parasitoids: a meta-analysis approach. Neotrop. Entomol. 47, 681-688. doi: 10.1007/s13744-017-0580-5

Mouden, S., Klinkhamer, P. G. L., Choi, Y. H., and Leiss, K. A. (2017). Towards ecofriendly crop protection: natural deep eutectic solvents and defensive secondary metabolites. Phytochem. Rev. 16, 935-951. doi: 10.1007/s11101-017-9502-8

Poreddy, S., Mitra, S., Schöttner, M., Chandran, J., Schneider, B., and Baldwin, I. T. (2015). Detoxification of hostplant's chemical defence rather than its anti-predator co-option drives $\beta$-glucosidase-mediated lepidopteran counter adaptation. Nat. Commun. 6:8525. doi: 10.1038/ncomms9525

Rivero, A., Magaud, A., Nicot, A., and Vezilier, J. (2011). Energetic cost of insecticide resistance in Culex pipiens mosquitoes. J. Med. Entomol. 48, 694700. doi: 10.1603/me10121

Roy, A., Walker, W. B., Vogel, H., Chattington, S., Larsson, M. C., and Anderson, P. (2016). Diet dependent metabolic responses in three generalist insect herbivores Spodoptera spp. Insect Biochem. Mol. Biol. 71, 91-105. doi: 10.1016/j.ibmb.2016. 02.006

Salunke, B. K., Kotkar, H. M., Mendki, P. S., Upasani, S. M., and Maheshwari, V. L. (2005). Efficacy of flavonoids in controlling Callosobruchus chinensis (L.) (Coleoptera: Bruchidae), a post-harvest pest of grain legumes. Crop Prot. 24, 888-893. doi: 10.1016/j.cropro.2005.01.013

Schwenke, R. A., Lazzaro, B. P., and Wolfner, M. F. (2016). Reproductionimmunity trade-offs in insects. Annu. Rev. Entomol. 61, 239-256. doi: 10.1146/ annurev-ento-010715-023924

Senthil-Nathan, S. (2013). Physiological and biochemical effect of neem and other Meliaceae plants secondary metabolites against Lepidopteran insects. Front. Physiol. 4:359. doi: 10.3389/fphys.2013.00359

Simmonds, M. S. (2003). Flavonoid-insect interactions: recent advances in our knowledge. Phytochemistry 64, 21-30. doi: 10.1016/s0031-9422(03)00293-0
Sun, W., Margam, V. M., Sun, L., Buczkowski, G., Bennett, G. W., Schemerhorn, B., et al. (2006). Genomewide analysis of phenobarbital-inducible genes in Drosophila melanogaster. Insect Mol. Biol. 15, 455-464. doi: 10.1111/j.13652583.2006.00662.x

Taggar, G. K., and Gill, R. S. (2016). Host plant resistance in Vigna sp. towards whitefly, Bemisia tabaci (Gennadius): a review. Entomol. Gen. 36, 1-24. doi: 10.1127/entomologia/2016/0184

Tang, F., Shao, Y., Yang, W., Yang, H., Li, P. P., Li, T. F., et al. (2013). Advance on insect strategy of expressing heat shock protein to response abiotic stress. Chin. Agric. Sci. Bull. 29, 181-184. doi: 10.11924/j.issn.1000-6850

Tangtrakulwanich, K., and Reddy, G. V. P. (2014). Advances in Plant Biopesticides. New Delhi: Springer.

Theis, N., and Lerdau, M. (2003). The evolution of function in plant secondary metabolites. Int. J. Plant Sci. 164, S93-S102. doi: 10.1086/37 4190

Ujvari, B., Casewell, N. R., Sunagar, K., Arbuckle, K., Wüster, W., Lo, N., et al. (2015). Widespread convergence in toxin resistance by predictable molecular evolution. Proc. Natl. Acad. Sci. U.S.A. 112, 11911-11916. doi: 10.1073/pnas. 1511706112

Valentino, J. B., and Harrelson, P. C. (2013). Phenotypic Plasticity: Molecular Mechanisms, Evolutionary Significance and Impact on Speciation. New York, NY: Nova Science Publishers.

West-Eberhard, M. J. (2003). Developmental Plasticity and Evolution. Oxford: Oxford University Press.

Whitman, D. W., and Ananthrakrishnan, T. N. (2009). Phenotypic Plasticity of Insects: Mechanisms and Consequences. Enfield, NH: Science Publishers.

Young, M. D., Wakefield, M. J., Smyth, G. K., and Oshlack, A. (2010). Gene ontology analysis for RNA-seq: accounting for selection bias. Genome Biol. 11:R14. doi: 10.1186/gb-2010-11-2-r14

Zhang, S., Pang, B., and Zhang, L. (2015). Novel odorant-binding proteins and their expression patterns in grasshopper, Oedaleus asiaticus. Biochem. Biophys. Res. Commun. 460, 274-280. doi: 10.1016/j.bbrc.2015. 03.024

Zhang, W. Z., He, B., Cao, G. C., Zhang, Z. H., Wu, Y. H., and Liu, S. C. (2013). Quantitative analysis of the effects of Stipa krylovii and Leymus chinensis on the factors of vatiability of Odaleous asiaticus. Acta Pratacult. Sin. 22, 302-309.

Zhen, Y., Aardema, M. L., Medina, E. M., Schumer, M., and Andolfatto, P. (2012). Parallel molecular evolution in an herbivore community. Science 337, 1634-1637. doi: 10.1126/science. 1226630

Conflict of Interest: The authors declare that the research was conducted in the absence of any commercial or financial relationships that could be construed as a potential conflict of interest.

Copyright (c) 2020 Huang, Lv, Zhang and Chang. This is an open-access article distributed under the terms of the Creative Commons Attribution License (CC BY). The use, distribution or reproduction in other forums is permitted, provided the original author(s) and the copyright owner(s) are credited and that the original publication in this journal is cited, in accordance with accepted academic practice. No use, distribution or reproduction is permitted which does not comply with these terms. 